[Radiocarbon, Vol. 16, No. 3, 1974, P. 307-330]

\title{
UNIVERSITY OF LUND RADIOCARBON DATES VII
}

\author{
SÖREN HÅKANSSON \\ Radiocarbon Dating Laboratory, Department of Quaternary Geology \\ University of Lund, Sweden
}

\section{INTRODUCTION}

Most of the ${ }^{14} \mathrm{C}$ measurements reported here were made between October 1972 and October 1973. Equipment, measurement, and treatment of samples are the same as reported previously $(\mathrm{R}, 1968, \mathrm{v} 10$, p 36-37; 1970, v 12, p 534).

Age calculations are based on a contemporary value equal to 0.950 of the activity of NBS oxalic acid standard and on the "conventional" half-life for ${ }^{14} \mathrm{C}$ of $5568 \mathrm{yr}$. Results are reported in years before 1950 (years $\mathrm{BP})$, and in the $\mathrm{AD} / \mathrm{BC}$ system. Errors quoted $( \pm 1 \sigma)$ include standard deviations of count rates for the unknown sample, contemporary standard, and background. Corrections for deviations from the "normal" ${ }^{13} \mathrm{C} /{ }^{12} \mathrm{C}$ ratio for terrestrial plants $\left(\delta^{13} \mathrm{G}=-25.0 \%\right.$ in the PDB scale) are applied for all samples; also for marine shells, because apparent age of recent marine shells is not always just counterbalanced by the effect of isotopic fractionation ( $c f$, Recent marine shells series, R, 1973, $\mathrm{v} 15, \mathrm{p} 506-507) . \delta^{13} \mathrm{C}$ values quoted are relative to the PDB standard.

The remark, "undersized; diluted", in Comments means the sample did not produce enough $\mathrm{CO}_{2}$ to fill the counter to normal pressure and "dead" $\mathrm{CO}_{2}$ from anthracite was introduced to make up the pressure. " ${ }^{\circ}$ " sample" indicates amount of $\mathrm{CO}_{2}$ derived from the sample present in the diluted counting gas; the rest is "dead" $\mathrm{CO}_{2}$. Organic carbon content reported for bone sample is calculated from yield of $\mathrm{CO}_{2}$ by combustion of pretreated collagen. Organic carbon lost during treatment is not included in calculated percentage.

The description of each sample is based on information provided by the submitter.

\section{ACKNOWLEDGMENTS}

The author thanks Kerstin Lundahl for sample preparations and routine operation of the dating equipment, and $\mathrm{R}$ Ryhage and his staff at the mass-spectrometric laboratory of Karolinska Inst, Stockholm, for the ${ }^{13} \mathrm{C}$ analyses. Special thanks are due Christian Cavallin for assistance with the electronic equipment.

\section{SAMPLE DESCRIPTIONS}

I. GEOLOGIC SAMPLES

\section{Malghult Pool series}

\section{A. Sweden}

Sediment (Livingstone core sampler, diam $60 \mathrm{~mm}$ ) from deepest part of Malghult Pool, Kristdala parish, S Sweden $\left(57^{\circ} 22^{\prime} \mathrm{N}, 16^{\circ} 15^{\prime} \mathrm{E}\right)$. Coll 1970 and subm by M Aronsson, Dept Quaternary Geol, Univ Lund. 
Dating is part of study on development of vegetation and cultural history in Kristdala area. Depths in sample titles refer to pool water level. Water depth $160 \mathrm{~cm}$ at sampling point. Pollen analyses by submitter. Only weak pretreatment with $\mathrm{HCl}$ due to small samples.

Lu-508. Malghult Pool 8, 299 to $306 \mathrm{~cm}$

Detritus gyttja. Low Picea value (ca $0.5 \%$ ).

Lu-509. Malghult Pool 7, 284 to $291 \mathrm{~cm}$

Detritus gyttja. Beginning of pronounced Picea increase.

Lu-510. Malghult Pool 6, 264 to $271 \mathrm{~cm}$

Detritus gyttja. Continued Picea increase.

\section{Lu-511. Malghult Pool 5, 244 to 251cm}

$$
\begin{gathered}
\mathbf{1 4 9 0} \pm \mathbf{5 0} \\
\operatorname{AD} 460 \\
\delta^{13} C=-30.6^{c} \%
\end{gathered}
$$

$$
1430 \pm 50
$$$$
\text { AD } 520
$$

$\delta^{13} \mathrm{C}=-28.4^{\mathrm{C} / \mathrm{CO}}$

$$
\begin{gathered}
\mathbf{1 1 8 0} \pm \mathbf{5 0} \\
\text { AD 770 } \\
\delta^{1 s} C=-29.1 / \% 0
\end{gathered}
$$

$$
920 \pm 55
$$$$
\text { AD } 1030
$$

$\delta^{13} \mathrm{C}=-28.9^{\circ} / \mathrm{co}$

Detritus gyttja. Just below Picea maximum (ca 12\%) combined with increase of cereal and weed pollen. Comment: undersized; diluted; $91 \%$ sample.

\section{Lu-512. Malghult Pool 4, 224 to 231em}

$$
730 \pm 55
$$

Detritus gyttja. Comment: undersized; diluted; $88 \%$ sample.

Lu-513. Malghult Pool 3, 223 to $230 \mathrm{~cm}$

$$
\begin{aligned}
& \mathbf{7 2 0} \pm \mathbf{5 0} \\
& \text { AD } 1230 \\
& \delta^{13} C=-29.7 \% / c
\end{aligned}
$$

Detritus gyttja. Comment: samples 3 and 4 are from adjacent cores.

\section{Lu-514. Malghult Pool 2, 198 to $209 \mathrm{~cm}$}

$$
\begin{gathered}
1290 \pm 80 \\
\text { AD 660 } \\
\delta^{13} C=-2 \delta .9 \%
\end{gathered}
$$

Detritus gyttja with lumps of another soil type containing littoral brown-mosses. Comment (MA): deviating age may be explained by presence of littoral plants, indicating temporary outflow of older littoral material and redeposition at sampling point. Undersized; diluted; $46 \%$ sample.

\section{Lu-515. Malghult Pool 1,184 to $191 \mathrm{~cm}$$$
610 \pm 65
$$$$
\text { AD } 1340
$$$$
\delta^{18} C=-30.6 \%
$$

Detritus gyttja. Comment: undersized; diluted; $56 \%$ sample. 


\section{Gothenburg Botanical Garden series}

Sediment from 2 cores from Gothenburg Botanical Garden (57 $\left.41^{\prime} 06^{\prime \prime} \mathrm{N}, 11^{\circ} 57^{\prime} 18^{\prime \prime} \mathrm{E}\right)$ were dated in conjunction with establishing a Pleistocene/Holocene boundary stratotype (Mörner, 1973). The Late glacial regression brought sea level down to ca $+15.5 \mathrm{~m}$, the Regression Max or ALV-1 shoreline of Mörner (1969), followed by Postglacial transgressions bringing sea level up to $+25.5 \mathrm{~m}$. Dates from Cores B 873 and B 870 relate to the ALV-1 stage and beginning of subsequent transgression. Numerous different analyses have been applied to Core B 873 (Mörner, 1973). Pretreated with HCl.

\section{Core B 873}

Foil piston core (diam $68 \mathrm{~mm}$ ) taken down to bedrock at $14.5 \mathrm{~m}$ depth. Surface at $+17.4 \mathrm{~m}$. Core was proposed as Pleistocene/Holocene boundary stratotype (Mörner, 1973). Coll 1970 and subm by N-A Mörner, Dept Geol, Univ Stockholm.

\section{Lu-552. Core B 873, 188.5 to $191.5 \mathrm{~cm}$}

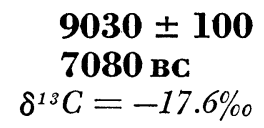

Gyttja. Early part of PTM-2 transgression. Comment: undersized; diluted; $86 \%$ sample.

Lu-553. Core B 873, 205 to $207 \mathrm{~cm}$

$9240 \pm 115$ $7290 \mathrm{BC}$

$\delta^{18} C=-19.1 \%$ o

Clayey gyttja. Early part of PTM-2 transgression. Comment: undersized; diluted; $72 \%$ sample.

\section{Lu-554. Core B 873, 229 to $231 \mathrm{~cm}$}

$9050 \pm 100$ $7100 \mathrm{BC}$

$\delta^{13} \mathrm{C}=-18.5 \%$ o

Gyttja. Earliest part of PTM-2 transgression. Comment: undersized; diluted; $90 \%$ sample.

\section{Lu-555. Core B 873, 251 to $255 \mathrm{~cm}$}

$9740 \pm 110$ $7790 \mathrm{BC}$

Clay with gyttja. Beginning of ALV-1 stage. Pollen Zone Boundary IV/V. Comment: undersized; diluted; $61 \%$ sample.

\section{Core B 870}

Surface at $+17.8 \mathrm{~m}, 40 \mathrm{~m}$ from Core B 873 . Samples from $15 \times 15 \mathrm{~cm}$ monolith cut from wall in dug out sec. Coll 1970 and subm by N-A Mörner.

\section{Lu-588. Core B 870, I, 176 to $177 \mathrm{~cm}$}

$$
\begin{gathered}
\mathbf{8 8 6 0} \pm 70 \\
6910 \mathrm{BC} \\
\delta^{13} \mathrm{C}=-18.7 \%
\end{gathered}
$$

Gyttja. Early part of PTM-2 transgression. Comment: undersized; diluted; $76 \%$ sample. (4 1-day counts.) 


\section{$7240 \mathrm{BC}$ \\ $\delta^{13} \mathrm{C}=-22.7^{C} / \mathrm{CO}$}

Gyttja. End of ALV-1 stage. Comment: undersized; diluted: 87,0 sample.

\section{Näckrosdammen series}

Limnic sediment from ancient lake Näckrosdammen, Änggården, Göteborg $\left(57^{\circ} 40^{\prime} \mathrm{N}, 11^{\circ} 57^{\prime} \mathrm{E}\right)$. Pollen-analytic investigation is part of study of Pleistocene/Holocene boundary (Berglund, 1973). Coll 1972 and subm by B E Berglund, Dept Quaternary Geol, Univ Lund. Depths given are below surface. Pretreated with HCl. All samples undersized; diluted.

\section{Lu-738. Näckrosdammen 1}

$$
\begin{gathered}
10,120 \pm 100 \\
8170 \mathrm{BC} \\
\delta^{13} C=-22.4 \% / c 0
\end{gathered}
$$

Fine detritus gyttja, 259 to $262 \mathrm{~cm}$. Pollen-analytically dated to transition zone Younger Dryas-Pre-Boreal. Comment: $77 \%$ sample. (3 1-day counts.)

\section{Lu-740. Näckrosdammen 3}

$$
\begin{gathered}
10,250 \pm 120 \\
8300 \mathrm{BC} \\
\delta^{13} C=-20.6 \%
\end{gathered}
$$

Muddy clay, 267 to $272 \mathrm{~cm}$. Pollen-analytically dated to the very end of Younger Dryas. Comment: 62\% sample. (3 1-day counts.)

\section{Lu-741. Näckrosdammen 4}

$$
\begin{gathered}
11,510 \pm 105 \\
9560 \mathrm{BC} \\
\delta^{1 s} C=-23.9 / \% 0
\end{gathered}
$$

Clay gyttja, 310 to $315 \mathrm{~cm}$. Pollen-analytically dated to end of Alleröd. Comment: $86 \%$ sample. (3 1-day counts.)

General Comment (BEB): dates of Samples 1 and 3 agree quite well with earlier dating of Pollen Zone Boundary Younger Dryas/Pre-Boreal. Sample 4 seems to be slightly older than expected.

\section{Southern Baltic, pine stump series}

Wood from pine stumps dredged by fishermen from bottom of $S$ Baltic Sea at water depth 48 and $57 \mathrm{~m}$. Coll by $\mathrm{H}$ Berntsson; subm by B E Berglund. Pretreated with $\mathrm{HCl}$ and $\mathrm{NaOH}$.

\section{Lu-702. Southern Baltic 2}

$$
\begin{gathered}
9480 \pm 95 \\
7530 \text { BC } \\
\delta^{1 s} C=-25.6 \% \%
\end{gathered}
$$

Wood from pine stump coll 1972 ENE of Stenshuvud $\left(55^{\circ} 42^{\prime} \mathrm{N}\right.$, $\left.14^{\circ} 34^{\prime} \mathrm{E}\right)$ at $48 \mathrm{~m}$ depth. 


\section{Lu-807. Southern Baltic 3}

Wood from pine stump coll $1973 \mathrm{SE}$ of Hanö $\left(55^{\circ} 49^{\prime} \mathrm{N}, 15^{\circ} 14^{\prime}\right.$ E) at $57 \mathrm{~m}$ depth.

General Comment (BEB): similar ages were obtained earlier for submerged pine stumps from bottom of Baltic ( $c f \mathrm{R}, 1972, \mathrm{v} 14, \mathrm{p} 386)$.

\section{Kullaberg series}

Polar bear femur from depression $\mathrm{E}$ of Kullagård, Kullaberg, NW Scania $\left(56^{\circ} 18^{\prime} \mathrm{N}, 12^{\circ} 29^{\prime} \mathrm{E}\right)$. Coll 1852 (Lindström, 1880, p. 4-5; Holst, $1902, p$ 11-12) and subm by B E Berglund. Bone from marl layer (Swedish märgel) underlain by gyttja. Bone dense and very well preserved. All samples undersized; diluted.

\section{Lu-660. Kullaberg, inner part}

$12,710 \pm 125$

$10,760 \mathrm{BC}$

Collagen from middle part of bone wall. Comment: organic carbon content: $4.5 \% ; 80 \%$ sample. (3 1-day counts.)

\section{Lu-661. Kullaberg, outer part \\ $12,740 \pm 170$ \\ $10,790 \mathrm{BC}$ \\ $\delta^{10} \mathrm{C}=-14.4 \%$}

Collagen from spongy and superficial material inside bone cavity. Comment: organic carbon content: $6.3 \% ; 56 \%$ sample. (3 1-day counts.)

\section{Lu-602. Kullaberg, intermediate part}

$$
\begin{gathered}
12,580 \pm 100 \\
10,630 \text { BC } \\
\delta^{13} C=-13.1 \%
\end{gathered}
$$

Collagen from bone material taken between inner and outer part. Comment: organic carbon content: $5.4 \%$; $86 \%$ sample. (2 2-day counts.) General Comment: agreement between dates indicates contamination is absent or insignificant. No corrections made for apparent age of bones of living polar bears (see Recent polar bears series, below).

\section{Recent polar bears series}

Radiocarbon activity of bone collagen from 2 recent pre-bomb polar bears was measured to find their apparent ages.

\section{Lu-715. Kapp Wijk}

$$
\begin{array}{r}
\text { Apparent age: } \mathbf{4 8 0} \pm \mathbf{7 0} \\
\delta^{13} \mathrm{C}=-14.6 \%
\end{array}
$$

Collagen from cranium of young polar bear coll 1959 at Kapp Wijk, Dickson Fiord, Spitsbergen $\left(78^{\circ} 30^{\prime} \mathrm{N}, 15^{\circ} 00^{\prime} \mathrm{E}\right)$ by Natascha Heintz, Paleontol Mus, Oslo, Norway; subm by $S$ Håkansson. Time elapsed since death of bear estimated from state of preservation of cranium at $50 \pm 50 \mathrm{yr}(\mathrm{BP})$. Activity measurement corrected for this time interval. 


\section{Lu-779. Kap Stephensen}

Collagen from humerus of ca 2-yr-old polar bear shot in 1932 outside Kap Stephensen, East Greenland (ca $68^{\circ} 25^{\prime} \mathrm{N}, 28^{\circ} 31^{\prime} \mathrm{W}$ ) by U M $\phi \mathrm{hl}$; subm by I S $\phi r e n s e n$, Zool Mus, Univ Copenhagen, Denmark. Activity measurement age-corrected for time between 1932 and 1950 .

\section{Växjösjön series}

Sediment from Lake Växjösjön at the town of Växjö, Central Småland $\left(56^{\circ} 52^{\prime} \mathrm{N}, 14^{\circ} 49^{\prime} \mathrm{E}\right)$. Coll $1972-73$ and subm by $\mathrm{G}$ Digerfeldt, Dept Quaternary Geol, Univ Lund. Dates were part of study of development of lake and surrounding landscape during latest ca $1500 \mathrm{yr}$. Dates to determine rate of sediment deposition. Depths refer to sediment surface. All samples consist of detritus gyttja.

\section{Lu-734. Växjösjön, 15 to $20 \mathrm{~cm}$}

Comment: pretreated with $\mathrm{HCl}$.

Lu-735. Växjösjön, 40 to $45 \mathrm{~cm}$

$760 \pm 50$

AD 1190

$\delta^{1 s} C=-26.0 \%$

$$
\begin{gathered}
\mathbf{1 1 2 0} \pm \mathbf{5 5} \\
\text { AD 830 } \\
\delta^{18} C=-26.0 \%
\end{gathered}
$$

Comment: mild pretreatment with $\mathrm{HCl}$ and $\mathrm{NaOH}$. Undersized; diluted; $91 \%$ sample.

Lu-736. Växjösjön, 65 to $70 \mathrm{~cm}$

Comment: same pretreatment as $\mathrm{Lu}-735$.

Lu-674. Växjösjön, 85 to $90 \mathrm{~cm}$

Comment: pretreated with HCl. $77 \%$ sample.

Lu-737. Växjösjön, 90 to $95 \mathrm{~cm}$

$$
920 \pm 50
$$$$
\text { AD } 1030
$$$$
\delta^{13} C=-26.3 \%
$$

AD 1160

$\delta^{13} C=-26.2 \%$ 。

$$
790 \pm 55
$$$$
\delta^{13} C=-26.2 \%
$$

AD 1320

$\delta^{1 s} \mathrm{C}=-27.1 \%$

Comment: mild pretreatment with $\mathrm{HCl}$ and $\mathrm{NaOH} .89 \%$ sample.

\section{Lu-675. Växjösjön, 105 to $110 \mathrm{~cm}$}

$640 \pm 55$ AD 1310

Comment: pretreated with $\mathrm{HCl} .77 \%$ sample.

\section{Lu-676. Växjösjön, 125 to $130 \mathrm{~cm}$}

$500 \pm 55$

AD 1450

$\delta^{13} C=-26.8 \%$

Comment: pretreated with $\mathrm{HCl} .67 \%$ sample. 
Lu-677. Växjösjön, 145 to $150 \mathrm{~cm}$

Comment: pretreated with $\mathrm{HCl} .75 \%$ sample.

\section{Lu-678. Växjösjön, 165 to $170 \mathrm{~cm}$}

Comment: pretreated with $\mathrm{HCl} .72 \%$ sample.

Lu-679. Växjösjön, 185 to $190 \mathrm{~cm}$

Just above strong increase of Juniperus. Comment: pretreated with HCl. $80 \%$ sample.

Lu-680. Växjösjön, 205 to $210 \mathrm{~cm}$

Comment: pretreated with HCl. $91 \%$ sample.

Lu-681. Växjösjön, 225 to $230 \mathrm{~cm}$

Comment: pretreated with $\mathrm{HCl}$.

Lu-682. Växjösjön, 245 to $250 \mathrm{~cm}$

Comment: pretreated with $\mathrm{HCl}$.

Lu-683. Växjösjön, 265 to $270 \mathrm{~cm}$

Comment: pretreated with $\mathrm{HCl} .93 \%$ sample.

Lu-856. Växjösjön, 285 to $290 \mathrm{~cm}$
$450 \pm 55$ AD 1500

$\delta^{13} C=-26.5 \%$

$$
480 \pm 55
$$

AD 1470

$\delta^{13} C=-26.7 \% / 60$

$\mathbf{4 4 0} \pm \mathbf{5 5}$
AD 1510
$\delta^{13} C=-26.7 \%$
pretreated with
$610 \pm 55$

AD 1340

$\delta^{13} C=-27.6 \%$

$$
\begin{array}{r}
\mathbf{7 7 0} \pm \mathbf{5 0} \\
\mathbf{A D} \mathbf{1 1 8 0} \\
\delta^{13} C=-28.2 \% \sigma
\end{array}
$$

$$
\begin{aligned}
& \mathbf{9 7 0} \pm \mathbf{5 0} \\
& \mathbf{A D} 980 \\
& \delta^{13} C=-28.0 \%
\end{aligned}
$$

$980 \pm 55$

AD 970

$\delta^{13} \mathrm{C}=-28.5 \%$

$1220 \pm 60$

AD 730

$\delta^{13} \mathrm{C}=-28.3 \%$

Comment: mild pretreatment with $\mathrm{HCl}$ and $\mathrm{NaOH} .75 \%$ sample.

\section{Lu-857. Växjösjön, 305 to $310 \mathrm{~cm}$}
$1340 \pm 60$ AD 610
$\delta^{13} C=-28.0 \%$

Slight increase of Juniperus. Comment: same pretreatment as Lu856. $82 \%$ sample.

\section{Lu-858. Växjösjön, 325 to $330 \mathrm{~cm}$}

$$
\begin{array}{r}
1400 \\
\operatorname{AD} 550
\end{array}
$$

$\delta^{13} \mathrm{C}=-28.3^{\prime \prime} / \mathrm{Co}$

Just above rational Picea limit. Comment: same pretreatment as Lu-856. $77 \%$ sample. 
Lu-859. Växjösjön, 345 to $350 \mathrm{~cm}$

$$
\begin{gathered}
\mathbf{1 3 7 0} \pm \mathbf{7 5} \\
\mathbf{A D} \mathbf{5 8 0} \\
\delta^{13} C=-28.6 \%
\end{gathered}
$$

Comment: same pretreatment as Lu-856. $50 \%$ sample.

General Comment (GD): upper samples (from ca $1.5 \mathrm{~m}$ and upwards) are disturbed and corresponding dates are erroneous due to redeposition of older sediment related to sediment dredging in lake during end of 19 th century and beginning of this century.

\section{Solingsmyran series}

Marine sediment from beneath peat bog Solingsmyran, Västmanland, Cientral Sweden $\left(59^{\circ} 50^{\prime} \mathrm{N}, 16^{\circ} 30^{\prime} \mathrm{E}\right)$. Coll 1971 and subm by $\mathrm{S}$ Welinder, Dept Quaternary Geol, Univ Lund. Depths given are below surface. Pretreated with $\mathrm{HCl}$.

Lu-718. Solingsmyran $1,2.44$ to $2.49 \mathrm{~m}$

Clayey mud.

Lu-719. Solingsmyran $2,2.93$ to $2.98 \mathrm{~m}$

Clayey mud.

$$
\begin{gathered}
\mathbf{6 2 7 0} \pm \mathbf{7 0} \\
\mathbf{4 3 2 0} \mathbf{\mathrm { BC }} \\
\delta^{13} C=-18.5 \% \\
\mathbf{6 4 5 0} \pm \mathbf{7 5} \\
\mathbf{4 5 0 0} \mathrm{BC} \\
\delta^{13} C=-15.9 \%
\end{gathered}
$$

\section{Rövallsmossen series}

Peat from the emerged bog Rövallsmossen, Västmanland, Central Sweden $\left(59^{\circ} 45^{\prime} \mathrm{N}, 16^{\circ} 17^{\prime} \mathrm{E}\right)$. Coll 1971 and subm by $\mathrm{S}$ Welinder. Depths are below surface. Pretreated with $\mathrm{HCl}$ and $\mathrm{NaOH}$.

\section{Lu-720. Rövallsmossen $1,0.45$ to $0.50 \mathrm{~m}$}

Sphagnum peat.

\section{Lu-721. Rövallsmossen $2,0.50$ to $0.55 \mathrm{~m}$}

Sphagnum peat.

$$
\begin{array}{r}
\mathbf{2 0 0} \pm \mathbf{5 0} \\
\text { AD } \mathbf{1 7 5 0} \\
\delta^{13} C=-22.0 \%
\end{array}
$$

AD 1170

$780 \pm 50$

$\delta^{13} C=-24.5 \%$

\section{Hasslöv series}

Samples from ca $15 \mathrm{~cm}$ peat underlain by gravel and overlain by sand at Hasslöv, S Halland $\left(56^{\circ} 25^{\prime} \mathrm{N}, 13^{\circ} 00^{\prime} \mathrm{E}\right)$. Coll 1972 and subm by H Svensson, Swedish Nat Sci Res Council, Stockholm.

\section{Lu-805. Hasslöv C, peat}

Upper part $(\mathrm{ca} 1 \mathrm{~cm})$ of peat. Depth $60 \mathrm{~cm}$ below surface. Comment: pretreated with $\mathrm{HCl}$ and $\mathrm{NaOH}$. 


\section{Lu-805A. Hasslöv C, humic acid}

$8600 \pm 90$

$6650 \mathrm{BC}$

$\delta^{13} \mathrm{C}=-27.0^{\mathrm{c} \%}$

Acid-precipitated part of $\mathrm{NaOH}$-soluble fraction from $\mathrm{Lu}-805$.

\section{Lu-805:2. Hasslöv C, new preparation}

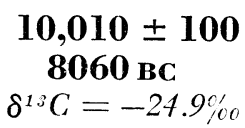

Remaining material from preparation for $\mathrm{Lu}-805$ was given stronger pretreatment with $\mathrm{NaOH}$ for removal of more humic acid.

Lu-806. Hasslöv D, peat

$2850 \pm 55$

$900 \mathrm{BC}$

$\delta^{13} \mathrm{C}=-27.0 \%$

Bottom part $(\mathrm{ca} 1 \mathrm{~cm})$ of peat. Depth $75 \mathrm{~cm}$ below surface. Comment: pretreated with $\mathrm{HCl}$ and $\mathrm{NaOH}$.

\section{Lu-806A. Hasslöv D, humic acid}

$$
\begin{gathered}
2400 \pm 55 \\
450 \mathrm{BC}
\end{gathered}
$$

Acid-precipitated part of a $\mathrm{NaOH}$-soluble fraction from $\mathrm{Lu}-806$.

\section{Lu-788. Lake Striern, Pinus stump}

$3680 \pm 60$

$1730 \mathrm{BC}$

$\delta^{13} \mathrm{C}=-24.8 \%$

Wood from stump (Pinus silvestris L), coll 1972 on land near W shore of Lake Striern, Östergötland $\left(58^{\circ} 05^{\prime} \mathrm{N}, 15^{\circ} 47^{\prime} \mathrm{E}\right)$ and subm by H Göransson, Dept Quaternary Geol, Univ Lund. For other dates from Lake Striern, see R, 1970, v 12, p 541-543. Pretreated with $\mathrm{HCl}$ and $\mathrm{NaOH}$. Comment (HG): stump was preserved by a lacustrine transgression, indicating climatic change. Shore with stumps became land by lowering of lake water level ca $100 \mathrm{yr}$ ago. Date as expected.

\section{Lake Vån series}

Sediment from Lake Vản, $6.3 \mathrm{~km}$ SE of Brokind RR Sta, Östergötland $\left(58^{\circ} 11^{\prime} \mathrm{N}, 15^{\circ} 47^{\prime} \mathrm{E}\right)$. Alt of lake: $+92.4 \mathrm{~m}$; size: ca $400 \times 400 \mathrm{~m}$. Coll 1969 and subm by H Göransson. Samples taken with $36 \mathrm{~mm}$ Livingstone core sampler, except Lu-814, which was aggregated from repeated samplings with Hiller sampler. All samples consist of fine detritus gyttja, somewhat clayey in lowest sample. Depths are below sediment-water interface. Water depth at sampling point, $3.8 \mathrm{~m}$. Dated as complement to Lake Striern series ( $\mathrm{R}, 1970, \mathrm{v} 12, \mathrm{p}$ 541-543). No pretreatment of Lu-822 and -823; all other samples pretreated with HCl.

\section{Lu-814. Vån, 420 to $430 \mathrm{~cm}$}

$9390 \pm 95$

Samples $85+86$. Rational Alnus limit.

\section{$7440 \mathrm{BC}$}

$\delta^{13} \mathrm{C}=-30.0 \%$ 
Lu-815. Vån, 370 to $380 \mathrm{~cm}$

Samples 75+76. Empiric Tilia limit.

Lu-816. Vån, 350 to $360 \mathrm{~cm}$

Samples $71+72$. Rational Tilia limit.

Lu-817. Vån, 265 to $275 \mathrm{~cm}$
$7800 \pm 80$

5850 вC

$\delta^{13} C=-30.9 \%$

$7170 \pm 80$

5220 BC

$\delta^{13} C=-29.4 \%$

$5960 \pm 70$

$4010 \mathrm{BC}$

$\delta^{13} C=-29.4 \%$

Samples 54+55. Classical Ulmus decline; rise of Alnus; 1st find of Triticum.

Lu-818. Vån, 235 to $245 \mathrm{~cm}$

$5640 \pm 70$

$3690 \mathrm{BC}$

$\delta^{18} C=-28.9 \%$

Samples $48+49$. Rise of Ulmus after decline; start of continuous Juniperus curve.

Lu-819. Vån, 165 to $175 \mathrm{~cm}$

$4800 \pm 65$

2850 BC

$\delta^{13} C=-28.2^{\mathrm{C}} / \% \mathrm{O}$

Samples $34+35$. Slightly decreasing Tilia in Pollen Zone SB 1.

\section{Lu-820. Vån, 140 to $150 \mathrm{~cm}$}

$4460 \pm 60$

2510 BC

$\delta^{13} \mathrm{C}=-30.8 \%$

Samples 29+30. Significant decline of Ulmus in SB 1.

\section{Lu-821. Vån, 60 to $70 \mathrm{~cm}$}

$3020 \pm 60$

$1070 \mathrm{BC}$

$\delta^{13} \mathrm{C}=-28.8^{\mathrm{C}} \%$

Samples $13+14$. Below empiric Picea limit; fairly high values of Plantago lanceolata and Rumex acetocella coll; below Secale limit. Comment: undersized; diluted; $89 \%$ sample.

\section{Lu-822. Vån, 30 to $40 \mathrm{~cm}$}

$$
\begin{gathered}
1410 \pm 60 \\
\text { AD 540 } \\
\delta^{13} C=-29.9 \%
\end{gathered}
$$

Samples $7+8$. Final decline of Quercetum Mixtum components. Comment: undersized; diluted; $75 \%$ sample.

\section{Lu-823. Vån, 10 to $20 \mathrm{~cm}$}

$$
\begin{gathered}
\mathbf{7 1 0} \pm 60 \\
\text { AD 1240 } \\
\delta^{13} C=-30.5 \%
\end{gathered}
$$

Samples $3+4$. Temporary decline of Picea indicating clearing; strong rise of Juniperus, Rumex acetocella coll, and Cerealea. Comment: undersized; diluted; $67 \%$ sample.

General Comment (HG): as Lake Vån is very small, it possibly dried out during part of Boreal time and perhaps also during other short 
periods. Sedimentation rate is very low between 430 and $350 \mathrm{~cm}$, and between $70 \mathrm{~cm}$ and surface layer, indicating possible hiata. Some characteristic levels (eg, rational Alnus limit; empiric Tilia limit) are distinctly older than in nearby lake Striern. Classical Ulmus decline seems older here than in other parts of Scandinavia.

\section{Lu-824. Hindbyhornet}

$$
\begin{gathered}
11,330 \pm 110 \\
9380 \mathrm{BC} \\
\delta^{13} \mathrm{C}=-20.3 \%
\end{gathered}
$$

Collagen from antler (Megaceros giganteus) from Late glacial sediment in small ancient lake at Hindby, Malmö $\left(55^{\circ} 35^{\prime} \mathrm{N}, 13^{\circ} 02^{\prime} \mathrm{E}\right)$. Coll 1972 by B Salomonsson; subm by R Liljegren, Dept Quaternary Geol, Univ Lund. Pollen investigation by submitter. Comment (RL): pollen study not yet completed, but preliminary results do not contradict date. Organic carbon content: $5.1 \%$.

\section{B. Norway}

\section{Lu-716. Domsängen, inner fraction}

$$
\begin{gathered}
11,260 \pm 110 \\
9310 \mathrm{BC} \\
\delta^{13} C=-1.0 \%
\end{gathered}
$$

Shells (Macoma calcarea) from sediment overlain by glaciofluvial material at Domsängen, ca $5 \mathrm{~km} \mathrm{NW}$ of Tönsberg, SE Norway $\left(59^{\circ} 18^{\prime}\right.$ $\mathrm{N}, 10^{\circ} 21^{\prime} \mathrm{E}$ ). Coll 1972 by $\mathrm{S}$ Håkansson. Date is important for chronology of $\mathrm{Ra}$ end moraine. Other dates connected with this moraine are summarized by Mangerud (1970, p 135). Many shell pairs were articulated when coll. Comment: inner fraction $(46 \%$ of shells) was used.

\section{Lu-7 17. Domsängen, outer fraction}

$$
\begin{gathered}
11,350 \pm 110 \\
9400 \mathrm{BC} \\
\delta^{13} \mathrm{C}=-1.3 \%
\end{gathered}
$$

Outer fraction of shells used for Lu-716. Comment: outer fraction was $43 \%$ of shells; outermost $11 \%$ removed by acid leaching.

General Comment: corrections for deviations from "normal" ${ }^{13} \mathrm{C} /{ }^{12} \mathrm{C}$ ratio for terrestrial plants $\left(\delta^{i s} C=-25.0 \%\right.$ in PDB scale) are applied also for shell samples. No corrections are made for apparent age of shells of living marine mollusks. Apparent age in area is studied by Mangerud (1972).

\section{Varanger Peninsula series}

Peat from different parts of old moraine line on Varanger Peninsula, N Norway. Studies in area by Svensson (1971a) and Svensson et al (1972). Coll 1972 by B Malmström and O Palmér, Dept Phys Geog, Univ Lund; subm by $\mathrm{H}$ Svensson.

\section{Lu-782. Torvvand, peat \\ $5540 \pm 70$ \\ 3590 BC \\ $\delta^{13} C=-27.8 \%$}

Sample I(1:1) from base of peat, overlying glacial clay at Torvvand 
$\left(70^{\circ} 28^{\prime} 20^{\prime \prime} \mathrm{N}, 18^{\circ} 19^{\prime} 15^{\prime \prime} \mathrm{E}\right)$. Comment: pretreated with $\mathrm{HCl}$ and $\mathrm{NaOH}$.

\section{Lu-782A. Torvvand, humic acid}

$$
\begin{gathered}
\mathbf{5 5 9 0} \pm \mathbf{7 0} \\
\mathbf{3 6 4 0} \mathbf{B C} \\
\delta^{13} \mathrm{C}=-27.8 \%
\end{gathered}
$$

Acid-precipitated part of $\mathrm{NaOH}$-soluble fraction from $\mathrm{Lu}-782$.

\section{Lu-709. Holmfjeldet, 3A+3B}

$7230 \pm 195$

5280 BC

$\delta^{13} C=-25.6 \%$

Samples 3A+3B from base of peat, overlying glacial clay at Holmfjeldet $\left(70^{\circ} 15^{\prime} \mathrm{N}, 19^{\circ} 40^{\prime} \mathrm{E}\right)$. Comment: no pretreatment; small sample; diluted; $31 \%$ sample.

\section{Lu-783. Holmfjeldet, II ( $1: 2)$}

$$
\begin{gathered}
\mathbf{8 0 5 0} \pm 85 \\
6100 \text { BC } \\
\delta^{13} C=-26.6 \%
\end{gathered}
$$

Sample II(1:2) from base of peat, overlying glacial clay at Holmfjeldet $\left(70^{\circ} 13^{\prime} 20^{\prime \prime} \mathrm{N}, 19^{\circ} 37^{\prime} 50^{\prime \prime} \mathrm{E}\right)$. Comment: mild pretreatment with $\mathrm{HCl}$ and $\mathrm{NaOH}$.

\section{Lu-784. Holmfjeldet, II $(2: 2)$}

$$
\begin{gathered}
\mathbf{6 8 0 0} \pm \mathbf{7 5} \\
\mathbf{4 8 5 0} \mathbf{B C} \\
\delta^{13} C=-26.3 \%
\end{gathered}
$$

Sample II(2:2) from upper part of base peat, underlying sand. Comment: mild pretreatment with $\mathrm{HCl}$ and $\mathrm{NaOH}$.

\section{Lu-785. Holmfjeldet, III (1:1)}

$$
\begin{gathered}
\mathbf{7 1 9 0} \pm \mathbf{8 0} \\
\mathbf{5 2 4 0} \mathbf{B C} \\
\delta^{13} C=-24.5 \%
\end{gathered}
$$

Sample III(1:1) from base of peat, overlying till at Holmfjeldet $\left(70^{\circ} 13^{\prime} 40^{\prime \prime} \mathrm{N}, 19^{\circ} 33^{\prime} 38^{\prime \prime} \mathrm{E}\right)$. Comment: mild pretreatment with $\mathrm{HCl}$ and $\mathrm{NaOH}$.

\section{Lu-786. Kobberhovedet, IV (1:1)}

$$
\begin{gathered}
5870 \pm 70 \\
3920 \text { BC } \\
\delta^{13} C=-27.5 \%
\end{gathered}
$$

Sample IV(1:1) from base of peat, overlying glacial clay. Comment: mild pretreatment with $\mathrm{HCl}$ and $\mathrm{NaOH}$.

\section{Spitsbergen}

\section{Lu-743. Advent Fiord}

$$
\begin{aligned}
& \mathbf{2 4 0} \pm \mathbf{5 0} \\
& \text { AD } \mathbf{1 7 1 0} \\
& \delta^{13} C=-23.8 \%
\end{aligned}
$$

Wood from tree from top surface of "Lagoon-pingo", in Moskus lagoon, inner part of Advent Fiord, Westspitsbergen $\left(78^{\circ} 14^{\prime} \mathrm{N}, 15^{\circ}\right.$ $45^{\prime}$ E). Coll 1972 by R Ahman; subm by H Svensson. Report of study in Advent Fiord area by submitter (Svensson, 1971b). For other date from same area, see $\mathrm{R}, 1970, \mathrm{v} 12, \mathrm{p} 546$. Pretreated with $\mathrm{HCl}$ and $\mathrm{NaOH}$. 


\section{Greenland}

\section{East Greenland series (III)}

Marine shells from emerged marine sediments in Kong Oscars Fjord - Vega Sund dist, Central East Greenland. Coll 1970 to 1972 and subm by C Hjort, Dept Quaternary Geol, Univ Lund; part of 3-yr study of ice oscillations and shoreline displacement. For other dates from area, see R, 1972, v 14, p 388-390; 1973, v 15, p 504-507. For apparent age of recent shells in area, see R, 1973, v 15, p 506-507 and Hjort (1973).

\section{Lu-710. Kap Biot}

$$
\begin{gathered}
\mathbf{9 8 2 0} \pm 95 \\
\mathbf{7 8 7 0} \mathbf{B C} \\
\delta^{13} C=+0.7 \% o
\end{gathered}
$$

Shells (Mya truncata, Hiatella arctica) from silt covering end moraine at Kong Oscars Fjord, side mouth of Edderfugledal $\left(72^{\circ} 56^{\prime} \mathrm{N}\right.$, $\left.22^{\circ} 40^{\prime} \mathrm{W}\right)$. Coll at $+40 \mathrm{~m}$.

\section{Lu-711. Lyells Land E}

$$
\begin{gathered}
8920 \pm 85 \\
6970 \text { BC } \\
\delta^{13} C=+0.1 \% o
\end{gathered}
$$

Shells (Mya truncata) from silt at +72 to $+85 \mathrm{~m}$ on E Lyells Land $\left(72^{\circ} 36^{\prime} \mathrm{N}, 24^{\circ} 44^{\prime} \mathrm{W}\right)$. Highest shell-bearing locality in this part of dist.

\section{Lu-712. Gudenelv}

$9980 \pm 95$ 8030 BC

$$
\delta^{13} \mathrm{C}=-0.2 \% \text { o }
$$

Shells (Hiatella arctica) from delta sediment at ca $+90 \mathrm{~m}$ (alt from map; only approx) at middle reaches of Gudenelv $\left(72^{\circ} 30^{\prime} \mathrm{N}, 23^{\circ} 05^{\prime}\right.$ W), interior of Traill ö. Highest shell-bearing deposit in this part of Traill ö.

\section{Lu-713. Rhedins Fjord}

$7310 \pm 85$

$5360 \mathrm{BC}$

$$
\delta^{13} C=+0.7 \%
$$

Shells (Mya truncata, Hiatella arctica, Macoma calcarea) from silt on inner side of end moraine in fjord valley. Coll at +20 to $+26 \mathrm{~m}$, and probably closely postdates ice retreat $\left(72^{\circ} 36^{\prime} \mathrm{N}, 26^{\circ} 23^{\prime} \mathrm{W}\right)$.

\section{Lu-714. Månedal, Sample 1}

$9070 \pm 90$

$7120 \mathrm{BC}$

$\delta^{13} C=+0.7 \%$

Shells (Mya truncata) from delta sediment at mouth of Månedal, $\mathrm{N}$ Traill $0 \ddot{0}\left(72^{\circ} 42^{\prime} \mathrm{N}, 22^{\circ} 58^{\prime} \mathrm{W}\right)$. Coll at +20 to $+25 \mathrm{~m}$, but delta surface at $+50 \mathrm{~m}$.

\section{Lu-764. Henrik Möller Dal}

$9880 \pm 95$ 7930 BC $\delta^{13} C=+0.2 \%$

Shells (Mya truncata, Hiatella arctica) from clay, covered by delta sediments, Henrik Möller Dal, Fleming Fjord ( $\left.71^{\circ} 53^{\prime} \mathrm{N}, 22^{\circ} 55^{\prime} \mathrm{W}\right)$. 
Coll at $+35 \mathrm{~m}$ but probably assoc with strandline $+60 \mathrm{~m}$, and closely dates ice retreat from outer Fleming Fjord.

\section{Lu-765. Murgangsdalen}

$$
\begin{gathered}
\mathbf{7 3 2 0} \pm \mathbf{7 5} \\
\mathbf{5 3 7 0} \mathbf{\text { BC }} \\
\delta^{13} C=-0.3 \%
\end{gathered}
$$

Shells (Mya truncata, Hiatella arctica, Macoma calcarea, Clinocardium ciliatum, and Mytilus edulis) from stony glaciomarine silt at $+10 \mathrm{~m}$ in outer part of Murgangsdalen, Kempe Fjord, Suess Land side $\left(72^{\circ} 55^{\prime} \mathrm{N}, 25^{\circ} 45^{\prime} \mathrm{W}\right)$. Probably a close date for deglaciation of outer part of valley.

\section{Lu-766. Kap Palander}

$\mathbf{8 4 2 0} \pm 80$

$6470 \mathrm{BC}$

$\delta^{13} C=+1.5 \%$

Shells (Hiatella arctica, Mya truncata, Nicania [Astarte] montagui) from silt at $+10 \mathrm{~m} \mathrm{~W}$ of Kap Palander, N Traill ö $\left(72^{\circ} 38^{\prime} \mathrm{N}, 22^{\circ} 41^{\prime} \mathrm{W}\right)$.

\section{Lu-767. Kap Hedlund}

$7860 \pm 80$

5910 вс

$\delta^{13} C=+0.6 \%$

Shells (Mya truncata, Hiatella arctica) from stony glaciomarine silty clay at $+40 \mathrm{~m}$, Kap Hedlund, Kempe Fjord $\left(72^{\circ} 44^{\prime} \mathrm{N}, 26^{\circ} 10^{\prime} \mathrm{W}\right)$. Highest shell-bearing sediment at locality, and probably deposited soon after ice retreat. $C f$ Noe-Nygaard (1932).

\section{Lu-768. Östernaesdeltat, Sample 1}

$9030 \pm 85$

$7080 \mathrm{BC}$

$\delta^{19} \mathrm{C}=+1.5 \%$

Shells (Mya truncata, Hiatella arctica, Tridonta [Astarte] borealis, Nicania [Astarte] montagui, Bathyarca [Arca] glacialis) from clay, overlain and underlain by sand and silt. Coll at $+22 \mathrm{~m}$ but deposited when sea level was higher than $+30 \mathrm{~m}$. Probably closely dates retreat of ice from terminal zone a few $\mathrm{km}$ inland. Delta $\mathrm{W}$ of Östernaes on $\mathrm{N}$ Traill ö $\left(72^{\circ} 49^{\prime} \mathrm{N}, 23^{\circ} 17^{\prime} \mathrm{W}\right)$.

\section{Lu-789. Antarctic Dal}

$$
9510 \pm 100
$$

$$
\begin{gathered}
7560 \text { BC } \\
\delta^{13} C=-0.2 \% \text { o }
\end{gathered}
$$

Shells (Mya truncata) from delta built up to ca $+50 \mathrm{~m}$. Coll at $+40 \mathrm{~m}$ at mouth of Majdal in Arctic Dal (Kolledal), Scoresby Land $\left(72^{\circ} 00^{\prime} \mathrm{N}\right.$, $23^{\circ} 20^{\prime} \mathrm{W}$ ). Comment: undersized; diluted; $85 \%$ sample.

\section{Lu-790. Kap Petersens}

$$
\begin{gathered}
9130 \pm 90 \\
7180 \mathrm{BC} \\
\delta^{1 s} C=-0.5 \%
\end{gathered}
$$

Shells (Mya truncata, Hiatella arctica, Macoma calcarea) from sandy sediment underlain by clay and overlain by gravelly subaerial sediment. Coll at +50 to $+55 \mathrm{~m}$; dates sea level around or somewhat above $+60 \mathrm{~m}$. Highest shell-bearing sediment at Kap Petersens, Kong Oscars Fjord $\left(72^{\circ} 25^{\prime} \mathrm{N}, 24^{\circ} 35^{\prime} \mathrm{W}\right)$. 


\section{Lu-791. Polhems Dal N, Sample 1}

$$
\begin{gathered}
7970 \pm 80 \\
6020 \mathrm{BC} \\
\delta^{13} \mathrm{C}=-0.8 \%
\end{gathered}
$$

Shells (Mya truncata, Hiatella arctica, Macoma calcarea) from sandy, silty bed underlain by varved clay ( $c f$ Lu-825, below) and clay; overlain by gravelly/sandy sediment. Coll at $+32 \mathrm{~m}$ but deposited when sea level was ca $+70 \mathrm{~m}$. N mouth of Polhemsdal, Lyells Land $\left(72^{\circ} 44^{\prime} \mathrm{N}, 25^{\circ}\right.$ $\left.05^{\prime} \mathrm{W}\right)$.

\section{Lu-792. Skipperdal}

$$
\begin{gathered}
8550 \pm 85 \\
6600 \mathrm{BC} \\
\delta^{13} \mathrm{C}=-1.1 \%
\end{gathered}
$$

Shells (Mya truncata, Hiatella arctica, Macoma calcarea) from clay underlying delta built up to $+60 \mathrm{~m}$ at mouth of Skipperdal, Segelsällskapets Fjord $\left(72^{\circ} 24^{\prime} \mathrm{N}, 24^{\circ} 55^{\prime} \mathrm{W}\right)$. Clay lies directly on striated and glacially sculptured rocks, and sample probably approximates deglaciation of this fjord. Coll at $+38 \mathrm{~m}$.

\section{Lu-793. Kap Laura}

$$
\begin{aligned}
& 8830 \pm 85 \\
& 6880 \mathrm{BC}
\end{aligned}
$$

Shells (Mya truncata) from delta built up to $+40 \mathrm{~m}$. Coll at $+20 \mathrm{~m}$. Kap Laura (name on Norwegian map 1:50.000), S Geog Soc Ö $\left(72^{\circ} 53^{\prime}\right.$ $\mathrm{N}, 23^{\circ} 25^{\prime}$ W). Cf Lu-646, R, 1973, v 15, p 505.

\section{Lu-825. Polhems Dal N, Sample 2}

Shells (Mya truncata, Hiatella arctica, Macoma calcarea) from varved clay at bottom of sequence described under Lu-791 above. Coll at $+24 \mathrm{~m}$. $\mathrm{N}$ mouth of Polhemsdal, Lyells Land $\left(72^{\circ} 44^{\prime} \mathrm{N}, 25^{\circ} 05^{\prime} \mathrm{W}\right)$.

\section{Lu-826. Holms Bugt, Sample 1}

$$
\begin{gathered}
8910 \pm 85 \\
6960 \text { BC } \\
\delta^{13} C=-0.2 \%
\end{gathered}
$$

Shells (Mya truncata, Hiatella arctica, Macoma calcarea), from delta built up to $+50 \mathrm{~m}$. Coll at $+45 \mathrm{~m}$. Holms Bugt, S Traill ö $\left(72^{\circ} 31^{\prime} \mathrm{N}\right.$, $\left.23^{\circ} 58^{\prime} \mathrm{W}\right)$.

\section{Lu-827. Holms Bugt, Sample 2}

Shells (Mya truncata, Hiatella arctica, Macoma calcarea, Nicania [Astarte] montagui) from delta sediment at $+15 \mathrm{~m}$. Probably dates shoreline at $+20 \mathrm{~m}$. Holms Bugt $\left(72^{\circ} 31^{\prime} \mathrm{N}, 23^{\circ} 58^{\prime} \mathrm{W}\right)$.

\section{Lu-828. Holms Bugt, Sample 3}

$$
\begin{gathered}
5570 \pm 65 \\
\mathbf{3 6 2 0} \mathbf{B C} \\
\delta^{13} C=-0.5 \%
\end{gathered}
$$

Shells (Mya truncata, Macoma calcarea, Serripes groenlandica, Clinocardium ciliatum) from sandy, silty sediment at $+8 \mathrm{~m}$. Probably dates 
shoreline at $+9 \mathrm{~m}$. Holms Bugt $\left(72^{\circ} 3 \mathrm{1}^{\prime} \mathrm{N}, 23^{\circ} 58^{\prime} \mathrm{W}\right)$. For other dates from Holms Bugt, see also Lu-489, -490, -529 (R, 1972, v 14, p 388-389).

\section{Lu-829. Månedal, Sample 2}

$8250 \pm 80$ $6300 \mathrm{BC}$

Shells (Mya truncata, Hiatella arctica, Tridonta [Astarte] borealis) from delta sediment at $+12 \mathrm{~m}$. Dates sea level at ca $+18 \mathrm{~m}$. Mytilus edulis and Chlamys [Pecten] islandicus occur in sediment but not in dated part of sample. Mouth of Månedal, N Traill ö $\left(72^{\circ} 42^{\prime} \mathrm{N}, 22^{\circ}\right.$ $\left.58^{\prime} \mathrm{W}\right)$. Cf Lu-714 above.

\section{Lu-830. Östernaesdeltat, Sample 2}

$$
\begin{gathered}
8270 \pm 80 \\
6320 \text { BC } \\
\delta^{13} C=-0.3 \%
\end{gathered}
$$

Shells (Mya truncata, Hiatella arctica, Macoma calcarea, Nicania [Astarte] montagui, Mytilus edulis) from sandy, silty sediment at $+30 \mathrm{~m}$. Delta W of östernaes on N Traill ö $\left(72^{\circ} 49^{\prime} \mathrm{N}, 23^{\circ} 17^{\prime} \mathrm{W}\right)$.

\section{Lu-831. Mestersvig}

$4130 \pm 60$

$2180 \mathrm{BC}$

Shells (Mya truncata, Hiatella arctica, Macoma calcarea, Clinocardium ciliatum) from delta foreset beds at $+4 \mathrm{~m}$. Predates sea level at $+6 \mathrm{~m}$. Mouth of Tunnelelv in Noret, Mestersvig $\left(72^{\circ} 13^{\prime} \mathrm{N}, 23^{\circ} 53^{\prime}\right.$ W). Cf Washburn and Stuiver (1962).

General Comment: corrections for deviations from "normal" ${ }^{13} \mathrm{C} /{ }^{12} \mathrm{C}$ ratio for terrestrial plants $\left(\delta^{13} C=-25.0 \%\right.$ in $\mathrm{PDB}$ scale) are applied also for shell samples. No corrections are made for apparent age of shells of living marine mollusks.

\section{E. Ireland}

\section{Blanket bog pine stump series}

Wood from stumps and root parts (Pinus siluestris L) from blanket bog areas in different parts of Ireland. Coll 1972 and subm by N Malmer, Dept Plant Ecol, Univ Lund. Studied for earliest possible beginning of blanket bog peat formation in those areas. Pretreated with $\mathrm{HCl}$ and $\mathrm{NaOH}$.

\section{Lu-772. Killarney}

$$
\begin{gathered}
4600 \pm 65 \\
2650 \mathrm{BC} \\
\delta^{13} C=-24.8 \%
\end{gathered}
$$

Wood from pine stump layer in contact zone between peat and underlying mineral soil, on $\mathrm{N}$ slope of Carrantoohill, $1 \mathrm{~km} \mathrm{~S}$ of small village Carrantoohill, $14 \mathrm{~km}$ WSW of Killarney, Co Kerry $\left(52^{\circ} 01^{\prime} \mathrm{N}\right.$, $\left.9^{\circ} 43^{\prime} \mathrm{W}\right)$. Alt $250 \mathrm{~m}$. Peat ca $1 \mathrm{~m}$ deep; highly humified. 


\section{Lu-773. Wicklow}

Wood from pine stump layer in contact zone between peat and underlying mineral soil, SE of TV sta ca $4 \mathrm{~km}$ SSW of Glencree, $20 \mathrm{~km}$ $\mathrm{S}$ of Dublin, Co Wicklow $\left(53^{\circ} 09^{\prime} \mathrm{N}, 6^{\circ} 17^{\prime} \mathrm{W}\right)$. Alt $630 \mathrm{~m}$ (Wicklow $\mathrm{mts})$. Peat ca $\mathrm{lm}$ deep; highly humified.

\section{Lu-774. Carrowmoore, Sample a}

$$
\begin{gathered}
4340 \pm 65 \\
2390 \text { BC } \\
\delta^{1 s} C=-25.0 \%
\end{gathered}
$$

Wood from pine stump layer in peat ca $20 \mathrm{~cm}$ above mineral soil, along rd from Glenamoy to Carrowmoore, $1 \mathrm{~km} \mathrm{~W}$ of Bellonaboy Bridge, Co Mayo $\left(54^{\circ} 14^{\prime} \mathrm{N}, 9^{\circ} 44^{\prime} \mathrm{W}\right)$. Alt $30 \mathrm{~m}$. Peat ca $1.2 \mathrm{~m}$ deep; highly humified.

\section{Lu-775. Carrowmoore, Sample b}

$7110 \pm 75$ $5160 \mathrm{BC}$ $\delta^{13} C=-25.6 \%$

Wood from pine root from mineral soil underlying blanket bog peat, same place as Lu-774. Root may be referred to pine stumps in contact zone between peat and underlying mineral soil. Charcoal found at this level.

\section{Leba series}

\section{F. Poland}

Charcoal from fossil soil humus layer, Leba Bay Bar, S coast of Baltic Sea, N Poland. Coll 1972 and subm by K Tobolski, Dept Plant Taxonomy and Phytogeog, A Mickiewicz Univ, Poznań, Poland. Fossil soils in area are described by Tobolski (1972a). Pretreated with $\mathrm{HCl}$ and $\mathrm{NaOH}$.

\section{Lu-761. Leba Bay Bar, Sample 3}

$$
\begin{array}{r}
\mathbf{1 5 4 0} \pm \mathbf{5 0} \\
\mathbf{A D} \mathbf{4 1 0} \\
\delta^{13} \mathrm{C}=-24.8 \% 0
\end{array}
$$

Pinus charcoal from lowest part of humus horizon in fossil podsol

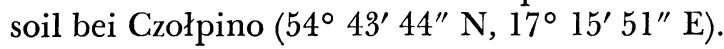

\section{Lu-762. Leba Bay Bar, Sample 17}

$$
\begin{gathered}
\mathbf{1 9 4 0} \pm \mathbf{5 0} \\
\mathbf{A D ~ 1 0} \\
\delta^{13} C=-24.5 \%
\end{gathered}
$$

Quercus charcoal from charcoal layer 1 to $2 \mathrm{~cm}$ thick underlain by sandy humus and overlain by raw humus in fossil soil horizon by LąskaDune $\left(54^{\circ} 45^{\prime} 09^{\prime \prime} \mathrm{N}, 17^{\circ} 25^{\prime} 16^{\prime \prime}\right.$ E). High percentages of Fagus-pollen above, and Quercus-pollen below charcoal layer.

\section{Lu-763. Ustka, Sample 31/71, insoluble}

$$
\begin{gathered}
11,800 \pm 115 \\
\mathbf{9 8 5 0} \mathbf{B C} \\
\delta^{13} C=-24.5 \%
\end{gathered}
$$

Insoluble part of organic matter from layer with tundra vegetation remnants from coastal cliff near Ustka $\left(54^{\circ} 35^{\prime} 53^{\prime \prime} \mathrm{N}, 16^{\circ} 54^{\prime} 11^{\prime \prime} \mathrm{E}\right)$. 
Coll 1972 and subm by $\mathrm{K}$ Tobolski. Cliff stratigraphy and fossil plants described by submitter (Tobolski, 1972b). Pretreated with HCl and $\mathrm{NaOH}$.

Lu-763A. Ustka, Sample 31/71, humic acid

$$
\begin{gathered}
11,850 \pm 115 \\
9900 \text { BC } \\
\delta^{1 s} C=-26.3 \%
\end{gathered}
$$

Acid-precipitated part of $\mathrm{NaOH}$-soluble fraction from $\mathrm{Lu}-763$. Comment: agreement between fractions indicates absence of contamination with younger humus.

$$
\text { II. ARCHAEOLOGIC SAMPLES }
$$

\section{Hagestad series}

Sweden

Charcoal from Hagestad, Löderup parish, Scania. Coll 1964 to 1972 and subm by $\mathbf{M}$ Strömberg, Hist Mus, Univ Lund. For other dates from Hagestad, see R, 1972, v 14, p 394-395; 1973, v 15, p 509. Pretreated with $\mathrm{HCl}$ and $\mathrm{NaOH}$.

\section{Lu-700. Hagestad 40 ${ }^{1}$, Sample 9}

Charcoal from trench with stone feature containing Bronze age finds, on field $S$ of rd Hagestaborg-Ramshög, Hagestad $40^{1}\left(55^{\circ} 24^{\prime} \mathrm{N}\right.$, 1409' E). Coll 1964.

\section{Lu-701. Hagestad 50 ${ }^{3}$, Sample 10}

$$
\begin{gathered}
\mathbf{3 2 7 0} \pm \mathbf{5 5} \\
\mathbf{1 3 2 0} \text { BC } \\
\delta^{1 s} C=-24.3 \%
\end{gathered}
$$

Charcoal from hearth on field $\mathrm{N}$ of brook, Hagestad $50^{3}\left(55^{\circ} 23^{\prime}\right.$ N, 14 09' E). Coll 1971. Assoc with Bronze age finds.

\section{Lu-781. Hagestad $44^{6}$ D, Sample 1}

$$
\begin{gathered}
\mathbf{1 8 3 0} \pm \mathbf{5 0} \\
\text { AD 120 } \\
\delta^{13} C=-24.5 \%
\end{gathered}
$$

Charcoal from hearth spatially connected with Late Neolithic as well as Roman Iron age features at Hagestad $44^{6} \mathrm{D}\left(55^{\circ} 23^{\prime} \mathrm{N}, 14^{\circ} 08^{\prime}\right.$ E). Coll 1972.

General Comment (MS): dates agree well with archaeol results based on artifact assemblage.

\section{Valleberga series}

Charcoal, wood, and bone from grave field at Valleberga, Scania $\left(55^{\circ} 24^{\prime} \mathrm{N}, 14^{\circ} 04^{\prime} \mathrm{E}\right)$. Coll Dec 1972 to March 1973 and subm by M Strömberg.

Lu-798. Valleberga 5

$$
\begin{gathered}
1990 \pm \mathbf{5 5} \\
\mathbf{4 0 ~ B C} \\
\delta^{13} C=-24.6 \%
\end{gathered}
$$

Charcoal from burnt post near urn grave at Valleberga 5. Com- 
ments: pretreated with $\mathrm{HCl}$ and $\mathrm{NaOH}$. (MS): somewhat later than expected.

\section{Lu-803. Valleberga $6^{7}$ B, Sample 10}

$3190 \pm 55$

1240 BC

$\delta^{13} C=-25.3 \%$

Wood fragments from remains of oak trunk coffin from Bronze age grave at Valleberga $6^{7} \mathrm{~B}$. Assoc with bronze objects from Period II. Comment: only weak pretreatment with $\mathrm{NaOH}$ due to poor state of preservation.

\section{Lu-804. Valleberga $6^{\tau}$ B, Sample 11}

$3170 \pm 55$

$1220 \mathrm{BC}$

$\delta^{13} \mathrm{C}=-18.6^{\mathrm{c}} \%$

Collagen from human bone from coffin dated as Lu-803. Comments: bone treated as described previously ( $R, 1970$, v 12, p 534). Organic carbon content: $5.5 \%$. (MS): agrees well with $\mathrm{Lu}-803$ and with time estimate based on assoc archaeol finds.

\section{Löderup series}

Charcoal from grave field at Löderup 15, Löderup parish, Scania (55 $23^{\prime} \mathrm{N}, 14^{\circ} 07^{\prime} \mathrm{E}$ ). Coll Dec 1972 to March 1973 and subm by $\mathrm{M}$ Strömberg. For other dates from Löderup 15, see R, 1973, v 15, p 509510. Pretreatment with $\mathrm{HCl}$ and $\mathrm{NaOH}$.

Lu-799. Löderup 15, Grave 75

$3360 \pm 60$

$1410 \mathrm{BC}$

Charcoal from bottom of Grave 75 (oak trunk grave).

$$
\delta^{13} \mathrm{C}=-24.1 \%
$$

\section{Lu-800. Löderup 15, Grave 60}

$2520 \pm 55$

Charcoal from hearth near Grave 60 (urn grave).

Lu-801. Löderup 15, Grave 91

$4130 \pm 60$

2180 BC

$\delta^{13} C=-25.0 \%$

Oak charcoal, id by $\mathrm{T}$ Bartholin, from Grave 91 (oak trunk grave). Assoc with flint axe.

\section{Lu-808. Löderup 15, Grave 100}

$3840 \pm 60$

Charcoal from Grave 100 (oak trunk grave).

$1890 \mathrm{BC}$

$$
\delta^{13} C=-25.6 \%
$$

\section{Lu-809. Löderup 15, Grave 92}

$4060 \pm 60$

2110 BC

Charcoal from pit near Grave 92. Assoc with arrowhead. 


\section{Lu-810. Löderup 15, Sample 8}

Charcoal from hearth in cultural layer $\mathrm{N}$ of Mound 3 on grave field. Late Bronze age pottery in adjacent cultural layer.

\section{Lu-811. Löderup 15, Grave 106}

$$
\begin{array}{r}
\mathbf{1 8 5 0} \pm \mathbf{5 0} \\
\text { AD 100 } \\
\delta^{13} \mathrm{C}=-24.7 \% \text { o }
\end{array}
$$

Charcoal from cremation burial at bottom of complex grave feature. Assoc with bone comb from Roman Iron age.

General Comment (MS): all dates agree well with results based on archaeol investigation.

\section{Gårdlösa series}

Charcoal from Gårdlösa, Smedstorp parish, SE Scania $\left(55^{\circ} 34^{\prime} \mathrm{N}\right.$, $14^{\circ} 08^{\prime}$ E). Coll 1971 and 1972 by A Nilsson; subm by B Stjernquist, Hist Mus, Univ Lund. Dated for study of continuity of Iron age settlement in Gårdlösa area. For other dates from area and references, see R, 1972, v 14, p 264-266, 392-393; 1973, v 15, p 510-511. Lu-708 only pretreated with $\mathrm{HCl}$ (small sample); all other samples pretreated with $\mathrm{HCl}$ and $\mathrm{NaOH}$.

\section{Lu-703. Gårdlösa 3, House LVIII}

$$
1570 \pm 50
$$

Charcoal from pit at bottom of house foundation.

\section{Lu-704. Gårdlösa $71^{1}$, Kiln 5}

Charcoal from mixed layer in remnants of kiln.

$$
\begin{gathered}
\mathbf{2 5 5 0} \pm \mathbf{5 5} \\
\mathbf{6 0 0} \text { BC } \\
\delta^{13} C=-24.7 \% \\
\mathbf{1 8 9 0} \pm \mathbf{5 5} \\
\mathbf{A D} \mathbf{6 0} \\
\delta^{13} C=-24.7 \%
\end{gathered}
$$

Lu-705. Gårdlösa $71^{1}$, Grave 97

Charcoal from Grave 97, just below plough-disturbed surface layer.

\section{Lu-706. Gårdlösa $71^{1}$, Hearth 194}

Charcoal from hearth.

\section{Lu-707. Gårdlösa $71^{1}$, house foundation}

Charcoal from hearth in $\mathrm{W}$ part of house foundation.

\section{Lu-708. Gårdlösa 10}

Charcoal from post-hole below stone pavement. Comment (BS):
$2480 \pm 55$

530 вC

$\delta^{13} C=-25.4 \%$

$2460 \pm 55$

$510 \mathrm{BC}$

$\delta^{13} C=-23.8 \%$ o

$$
\begin{aligned}
& \mathbf{3 4 0} \pm \mathbf{6 0} \\
& \text { AD 1610 } \\
& \delta^{13} C=-25.4 \%
\end{aligned}
$$


unexpected young date; charcoal apparently not contemporaneous with stone feature.

General Comment (BS): all dates except Lu-708 agree well with estimates based on archaeol material.

\section{Slädö Ship series}

Oak wood from ancient ship on bottom of strait at Slädö I, Listerby, Blekinge $\left(56^{\circ} 04^{\prime} \mathrm{N}, 15^{\circ} 25^{\prime} \mathrm{E}\right)$. Ship was said to be from Viking age. Coll by Blekinge Mus; subm by B E Berglund. Pretreated with $\mathrm{HCl}$ and $\mathrm{NaOH}$.

\section{Lu-744. Slädö Ship 1}

$$
\begin{array}{r}
\mathbf{2 9 0} \pm \mathbf{5 0} \\
\text { AD } 1 \mathbf{6 6 0} \\
\delta^{13} C=-23.8 \%
\end{array}
$$

Wood, probably from ship frame. Coll 1941. Object K 5070.

\section{Lu-745. Slädö Ship 2}

$$
\begin{array}{r}
\mathbf{5 9 0} \pm \mathbf{5 0} \\
\text { AD 1360 } \\
\delta^{13} C=-24.8 \%
\end{array}
$$

Small piece of wood, origin unknown. Object B1. M. 16111:4.

General Comment (BEB): ship seems much younger than expected; probably not $>300$ yr old

\section{Östanön-Kvalmsö-Helgeö series}

Wood from artificial blocking at 2 to $3 \mathrm{~m}$ depth in strait named Kålfjärden between is. Östanön, Helgeö, and Kvalmsö, Listerby, Blekinge $\left(56^{\circ} 10^{\prime} \mathrm{N}, 15^{\circ} 25^{\prime} \mathrm{E}\right)$. Coll 1972 by Blekinge Mus; subm by $\mathrm{B} \mathrm{E}$ Berg. lund. Other dates from similar blockings reported previously (R, 1968, v 10, p 50; 1969, v 11, p 448-449; 1972, v 14, p 397-398). Pretreated with $\mathrm{HCl}$ and $\mathrm{NaOH}$.

\section{Lu-769. Östanön 2}

$$
\begin{gathered}
\mathbf{9 4 0} \pm \mathbf{5 0} \\
\text { AD 1010 } \\
\delta^{13} C=-26.6 \%
\end{gathered}
$$

Wood and bark from huge ash pile standing in mud.

\section{Lu-770. Kvalmsö 5}

Wood from ash pile.

$$
\begin{gathered}
\mathbf{1 0 5 0} \pm \mathbf{5 0} \\
\mathbf{A D} 900 \\
\delta^{13} C=-25.4 \%
\end{gathered}
$$

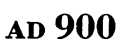

\section{Lu-771. Helgeö}

$$
\begin{gathered}
\mathbf{9 6 0} \pm \mathbf{5 0} \\
\text { AD 990 } \\
\delta^{13} C=-27.7 \% 0
\end{gathered}
$$

Wood from alder pile.

General Comment (BEB): new dates confirm older ones from $\mathrm{E}$ part of this archipelago, $i e$, blockings apparently built ca AD 1000 .

\section{Norrvidinge series}

Charcoal from buildings from Late Neolithic to Early Bronze age at Norrvidinge, Scania $\left(55^{\circ} 51^{\prime} \mathrm{N}, 13^{\circ} 06^{\prime} \mathrm{E}\right)$. Coll 1971 and subm by 
J Callmer, Hist Mus, Univ Lund. Preliminary report by submitter (Callmer, 1973).

Lu-836. Norrvidinge $3^{22-23}$, Feature 338

$3620 \pm 135$

$1670 \mathrm{BC}$

$\delta^{13} C=-25.5 \%$

Charcoal from post-hole in pit-dwelling (Feature 338). Assoc with flint and pottery. Comment: no pretreatment (small sample).

Lu-837. Norrvidinge $3^{22-23}$, Feature 339:8

$2960 \pm 55$

1010 BC

$\delta^{13} C=-23.0 \%$

Charcoal from Sec 8 of pit-dwelling (Feature 339). Assoc with daub, fint, and pottery.

General Comment (JC): agrees fairly well with archaeol date.

\section{Löddesborg series}

Charcoal from settlement area at Löddesborg, Scania $\left(55^{\circ} 45^{\prime} \mathrm{N}\right.$, $\left.12^{\circ} 59^{\prime} \mathrm{E}\right)$. For other date from Löddesborg, see R, 1973, v 15, p 508. Site is culturally closely connected to area studied at Norrvidinge (above). Coll 1972 to 1973 and subm by J Callmer. Pretreated with $\mathrm{HCl}$ and $\mathrm{NaOH}$.

Lu-838. Fårabacken, Löddesborg, House 1

$3440 \pm 55$

$1490 \mathrm{BC}$

$\delta^{13} C=-24.2 \%$

Charcoal from bottom layer in House 1. Assoc with flint and pottery (Late Neolithic to Early Bronze age).

\section{Lu-839. Firabacken, Löddesborg, Feature I-73 $3720 \pm 60$

Oak charcoal, id by $\mathrm{T}$ Bartholin, from $0.5 \mathrm{~m}$ below surface in pit with Middle Neolithic pottery and flint (Battle-axe culture).

General Comment (JC): agrees fairly well with archaeol date.

\section{Lu-742. Näsums Gudahage}

$$
\begin{array}{r}
\mathbf{6 1 0} \pm \mathbf{7 0} \\
\text { AD 1340 } \\
\delta^{1 s} C=-21.2 \%
\end{array}
$$

Collagen from part of poorly preserved human skull from grave at Gudahagen, Näsum parish, Scania $\left(56^{\circ} 09^{\prime} 55^{\prime \prime} \mathrm{N}, 14^{\circ} 30^{\prime} \mathrm{E}\right)$. Skull from gravel $0.6 \mathrm{~m}$ below surface. Coll 1972 by N-G Larsson; subm by M P Malmer, Hist Mus, Univ Lund. For other date from this site, see R, 1969, v 11, p 449. Comment: expected age 550 to 950 yr. Sample undersized; diluted; $51 \%$ sample. Organic carbon content: $1.4 \%$.

\section{Rogen series (II)}

III. MODERN PLANT SAMPLES

Samples from thick carpets of lichen (Cladonia alpestris) growing in open $\mathrm{mt}$ region above tree line, alt $900 \mathrm{~m}$, at Lake Rogen, Härjedalen 
$\left(62^{\circ} 30^{\prime} \mathrm{N}, 12^{\circ} 30^{\prime} \mathrm{E}\right)$. Coll 1964, 1968, and 1970 by Lidén, Persson, and Mattsson; subm by S Mattsson, Radiation Phys Dept, Univ Lund. First part of Rogen series pub previously (R, 1972, v 14, p 399). See also comprehensive report by submitter (Mattsson, 1972). All samples pretreated with $\mathrm{HCl}$.

Results are given as a difference, $\Delta$, from our radiocarbon standard (95\% activity of NBS oxalic acid standard, age corrected to 1950):

$$
\Delta=\delta^{14} \mathrm{C}-\left(2 \delta^{13} \mathrm{C}+50\right)\left(1+\frac{\delta^{14} \mathrm{C}}{1000}\right)
$$

where $\delta^{14} \mathrm{C}$ is observed deviation from radiocarbon standard in per mil and $\delta^{13} \mathrm{C}$ deviation from PDB standard in per mil.

\section{Lu-723. Rogen, V 532, 1964}

$$
\begin{aligned}
& \Delta=142 \pm 6 \% \\
& \delta^{13} C=-22.0 \%
\end{aligned}
$$

Unfractionated sample (Cladonia alpestris). Coll Sept 29, 1964.

$$
\begin{array}{lll}
\text { Lu-724. Rogen, V 786, } 1968 & \Delta=184 \pm 6 \% \\
& & \delta^{13} C=-22.2 \%
\end{array}
$$

Unfractionated sample( Cladonia alpestris). Coll Oct 1, 1968.

$$
\begin{array}{lll}
\text { Lu-725. Rogen, V } 919 \text { A, } 1970 & \Delta=\mathbf{5 8 4} \pm \mathbf{7} \% 0 \\
& & \delta^{18} C=-21.5 \% \circ
\end{array}
$$

Top fraction, 0 to 3cm (Cladonia alpestris). Coll Sept 28, 1970.

$$
\begin{array}{lll}
\text { Lu-726. Rogen, V } 919 \text { B, } 1970 & \Delta=\mathbf{3 7 1} \pm 7 \% \\
\delta^{13} C=-21.2 \%
\end{array}
$$

Middle fraction, 3 to $6 \mathrm{~cm}$ from top (Cladonia alpestris).

$$
\text { Lu-727. Rogen, V } 919 \text { C, } 1970 \quad \Delta=101 \pm 6 \%
$$

Lower fraction, below $6 \mathrm{~cm}$ from top (Cladonia alpesteris).

$$
\begin{array}{lr}
\text { Lu-728. Rogen, V } 919 \text { D, } 1970 & \begin{array}{c}
\Delta=\mathbf{2 7} \\
\delta^{13} C=-22.4 \% \circ
\end{array}
\end{array}
$$

Bottom fraction (gelatinous layer and decomposed lichen material; cf Mattsson, 1972, p 8-9).
Lu-729. Rogen, V 927 A, 1970
$\Delta=613 \pm 8 \%$ $\delta^{13} C=-22.4 \%$

Top fraction, 0 to 3cm (Cladonia alpestris). Coll. Sept 29, 1970.
Lu-730. Rogen, V 927 B, 1970
$\Delta=\mathbf{3 4 7} \pm \mathbf{7} \%$
$\delta^{1 s} C=-21.8 \%$

Middle fraction, 3 to $6 \mathrm{~cm}$ from top (Cladonia alpestris).
Lu-731. Rogen, V 927 C, 1970
$\Delta=59 \pm 6 \%$ $\delta^{1 s} C=-21.7 \%$

Lower fraction, below $6 \mathrm{~cm}$ from top (Cladonia alpestris). 


\section{Lu-732. Rogen, V 927 D, $1970 \quad \Delta=-1 \pm 6 \%$ \\ $\delta^{13} C=-22.1 \%$}

Bottom fraction (gelatinous layer and decomposed lichen material).

REFERENCES

Berglund, B E, 1973, Pollen analysis of Core B 873 and an adjacent lacustrine section, in: Mörner, N-A (ed), The Pleistocene/Holocene boundary. A proposed boundarystratotype in Gothenburg, Sweden, $\mathrm{p}$ 74-81.

Callmer, J, 1973, Preliminary report on a complex of buildings from the Late Neolithic-Early Bronze Age at Norrvidinge, Scania: Lunds Univ Hist Mus Medd 1971-72, p 120-143.

Håkansson, Sören, 1970, University of Lund radiocarbon dates III: Radiocarbon, v 12, p 534-552. $\frac{\mathrm{p} 534-552 .}{380-400}$. 1972 , University of Lund radiocarbon dates $\mathrm{V}$ : Radiocarbon, v 14, $\mathrm{p}$ 493-513.

Hjort, C, 1973, A sea correction for East Greenland: Geol Fören Stockholm Förh, v $95, \mathrm{p} 132-134$

Holst, N O, 1902, Några subfossila björnfynd: Sver Geol Unders, ser C, no. 189.

Lindström, Axel, 1880, Beskrifning till Kartbladen Kullen och Höganäs: Sver Geol Unders, ser Aa, no. 77 \& 78.

Mangerud, Jan, 1970, Late Weichselian vegetation and ice-front oscillations in the Bergen district, Western Norway: Norsk geog tidsskr, v 24, p 121-148.

1972, Radiocarbon dating of marine shells, including a discussion of apparent age of recent shells from Norway: Boreas (Oslo), v 1, p 143-172.

Mattsson, Sören, 1972, Radionuclides in lichen, reindeer and man: Thesis, Fac Math \& Nat Sci, Univ Lund.

Mörner, N-A, 1969, The Late Quaternary history of the Kattegatt Sea and the Swedish west coast; deglaciation, shorelevel displacement, chronology, isostasy and eustasy: Sver Geol Unders, ser C, no. 640, 487 p. (ed), 1973, The Pleistocene/Holocene boundary. A proposed boundarystratotype in Gothenburg, Sweden: mimeographed, $163 \mathrm{p}$

Noe-Nygaard, A, 1932, Remarks on Mytilus edulis in raised beaches in East Greenland: Grönland Medd, v 95, no. 2, 23 p.

Svensson, Harald, 1971a, Några drag i Varangerhalvöns geomorfologi i belysning av nya flygfotografier: Svensk Geog Årsb, v 47, p 7-28. p 168-174.

Svensson, H, et al, 1972, Nordvarangermoränen-en preliminär rapport: Lunds Lniv Naturgeog Inst, Rapporter och Notiser, no. 13.

Tobolski, K, 1972a, The fossil soils of the Leba Bay Bar, in: Galon, R (ed), internat conf INQUA subcommission on shorelines of northwestern Europe, Sopot 1972, Guide-Book of the Excursions, p 37-39. p 41.44

Washburn, A L and Stuiver, Minze, 1962, Radiocarbon-dated postglacial delevelling in Northeast Greenland and its implications: Arctic, v 15, p 66-73. 